\title{
Frequency Dependence of $D$-Region Scattering at VHF
}

\author{
J. C. Blair, R. M. Davis, Jr., and R. C. Kirby \\ Contribution from Central Radio Propagation Laboratory, National Bureau of Standards, Boulder, Colo.
}

(Received March 30, 1961)

\begin{abstract}
Results are given of a one-year program of observation of frequency dependence of system loss for $D$-region scattering at VHF. Continuous simultaneous observations were made at five frequencies from 30 to $108 \mathrm{Mc} / \mathrm{s}$, using narrow beam antennas scaled in dimensions and height according to wavelength; the path was from Long Branch, Ill., to Boulder, Colo., a distance of $1,300 \mathrm{~km}$. Hourly values of system loss closely followed the relationship $p_{b} / p_{a} \propto f^{n}$. The value of $n$ varied with time of day and season, with 90 percent of all observations lying in the range 7 to $9 \frac{1}{2}$ for scaled antenna systems. Diurnal and seasonal variation of $n$ are attributed to changing relative roles of turbulence and meteoric reflections. Effects of normal ionospheric absorption are not apparent. A special analysis is made of frequency dependence during weak signal conditions. Results are also given of studies of the frequency dependence of fading characteristics, the effect of beamwidth, and SID behavior.
\end{abstract}

\section{Introduction}

Continuous radio-wave scattering at very high frequencies, from turbulent irregularities and meteoric ionization in the $D$-region of the ionosphere, has been intensively investigated [Bailey, Bateman and Kirby, 1955] since 1951, and has been exploited for communication systems in several areas of the world [JTAC, 1960]. This paper reports an experimental study of the frequency dependence in the range 30 to $108 \mathrm{Mc} / \mathrm{s}$. One of the authors has already published preliminary data [Blair, 1959].

Frequency dependence is of considerable engineering importance in the design of ionosphericscatter communication systems. The higher the frequency one can use, the greater the freedom from interference, especially that propagated to great distances by the F2-layer and sporadic- $E$ ionization. To raise the frequency, however, increases propagation losses until, in spite of the accompanying decrease of the background level of galactic noise, excessive transmitter power is required for a needed transmission capacity. For design purposes one is especially interested in the frequency dependence characteristic of weak-signal conditions.

Interpretation of the physics of the scattering process also requires knowledge of the frequency dependence. In studying the role of turbulence, under the usual assumptions of single isotropic scattering at an average angle $\theta$, observations of frequency dependence serve to evaluate the spectrum of sizes of irregularities, $S(k)$. The scattering process acts like a narrow band filter, emphasizing the sizes of irregularities which have wavenumbers equal to

$$
k=\frac{4 \pi}{\lambda} \sin \frac{\theta}{2}
$$

where

$\lambda=$ wavelength

$\theta=$ scattering angle, shown in figure 1 .
Wheelon [1960a] has recently summarized turbulence theory for $D$-region scatter. He treats the single scattering description of electromagnetic response of electron density irregularities in time and space, and discusses several theories for turbulent mixing of electron density.

Neglecting absorption, the total received power in VHF scatter propagation is the resultant of components due to turbulent scattering, meteor reflections, and noise, which may be expressed as a function of frequency:

$$
p_{a}(f)=p_{s}(f)+p_{m}(f)+p_{N}(f)
$$

where

$p_{a}=$ available power at terminal of receiving antenna $p_{s}=$ component of available power from scattering from turbulent irregularities

$p_{m}=$ component of available power from meteor reflections

$p_{N}=$ component of available power from external noise usually mainly galactic

$f=$ carrier frequency, megacycles per second

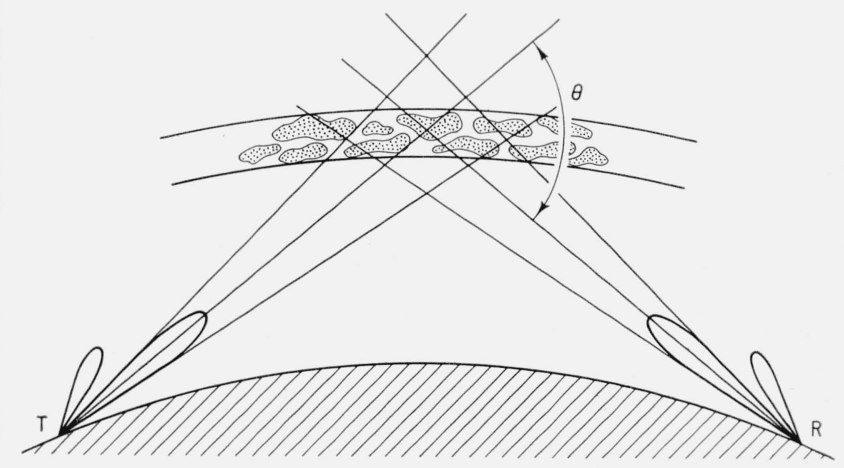

Figure 1. Geometry of D-region scattering. 
Other modes which sometimes occur, such as $E_{s}$, auroral echoes, F2-propagated ground backscatter, are recognizable by the abnormal character of the recording, and such data are not used in the analysis of frequency dependence. Adequate power is used in the experiment to keep the signal-to-noise ratio large, usually greater than 10 , so that $p_{N}$ can be neglected. The effect of absorption can be indicated:

$$
\begin{aligned}
p_{a}(f)=p_{s}(f) \exp \left[\frac{-2}{\left(f+f_{H}\right)^{2}} \int_{0}^{h_{s}} \alpha \sec \theta d h\right] \\
+p_{m}(f) \exp \left[\frac{-2}{\left(f+f_{H}\right)^{2}} \int_{0}^{h_{m}} \alpha \sec \theta d h\right]
\end{aligned}
$$

where

$f_{H}$ is the gyrofrequency, megacycles per second $\alpha$ is absorption coefficient, per element thickness, $d h$, of absorbing region

$h_{s}$ is the effective scattering height

$h_{m}$ is the height of meteoric reflection

for the oblique transmission through the absorbing region enroute to and from the scattering region or meteor reflection.

This is the first published report of comprehensive results of simultaneous observations at a series of frequencies, rather than a pair at a time, throughout the range of interest. The frequency dependence is expressed in terms of $n$ in the relation

$$
p_{t} / p_{a} \propto f^{n}
$$

where

$$
\begin{aligned}
p_{t} & =\text { power to transmitting antenna } \\
p_{a} & =\text { available power at receiving antenna } \\
f & =\text { frequency }
\end{aligned}
$$

Earlier experiments showed a variation of $n$. The Bailey, Bateman and Kirby [1955] measurements, obtained in two nonsimultaneous tests, indicated lower values of $n$ in the frequency range below 50 $\mathrm{Mc} / \mathrm{s}$ than in the range above $50 \mathrm{Mc} / \mathrm{s}$. This led Wheelon [1957] to suggest a theoretical explanation for a dichotomy in the scaling law; he emphasized at the same time the need of carefully scaled simultaneous measurements over the whole frequency range.

Some interpretation is given in this report of the variation in relative contribution of meteoric reflections and turbulent scattering, and the role of absorption.

\section{Experimental Conditions}

System loss, $p_{t} / p_{a}$, has been compared at a series of frequencies, using a fixed path length. The angle $\theta$ is nearly constant; diurnal and seasonal changes in height of scattering introduce slight variations.

Identical antenna patterns and gains are preserved at all frequencies by scaling antenna dimensions and height. Thus, the scattering volume is illuminated and viewed uniformly throughout the frequency range. As a consequence of scaling antennas, an extra power of 2 is contained in the exponent, $n$, for system loss, because the aperture of the receiving antenna varies as $\lambda^{2}$. One must subtract a power of 2 from the values of $n$ (divide system loss by $f^{2}$ ) for scaled antennas given in this paper, ${ }^{1}$ to make comparison with theoretical frequency dependence of scattering cross section $\sigma$, or with $S(k)$ values.

Figure 2 shows the geographical layout of the experimental path. Continuous transmissions were maintained at five frequencies from near $30 \mathrm{Mc} / \mathrm{s}$ to near $108 \mathrm{Mc} / \mathrm{s}$. Narrow-beam rhombic antennas were used, scaled in dimensions and height; the leg length was 25 wavelengths, one-half the obtuse angle was $80.2^{\circ}$ and the horizontal half-power beamwidth was $6^{\circ}$. The elevation of the axes of the main lobes was $4.6^{\circ}$; the beams were directed to intersect at the path midpoint at an ionospheric height of 85 $\mathrm{km}$. Losses in transmitting and receiving transmission line systems were measured, and observed data at each frequency corrected accordingly. Finally, aircraft measurements of antenna gains and radiation patterns were carried out. The average value of gain was $18.8 \mathrm{db}$ relative to a halfwave reference dipole at the same height; measured values of gain for all antennas were found to be within $\pm 0.8 \mathrm{db}$ of the average value. The sites were nearly ideally flat over at least the first Fresnel zone for ground reflection, and the aircraft measurements of radiation patterns confirmed the uniformity of the main lobes at all frequencies.

Transmitter power supplied to the antennas ranged from a nominal value of $2 \mathrm{kw}$ at $30 \mathrm{Mc} / \mathrm{s}$ to $20 \mathrm{kw}$ at $108 \mathrm{Mc} / \mathrm{s}$; the power at each frequency was calibrated and records were kept so that the received signal intensity could be adjusted to a standard reference level. Receiver noise bandwidths were 300 $\mathrm{c} / \mathrm{s}$ at all frequencies except $108 \mathrm{Mc} / \mathrm{s}$, for which a $25 \mathrm{c} / \mathrm{s}$ band was used.

1 This use of $n$ is at variance with its use in the references, including previous publications of the authors; it has been adopted because of the convenience of publications of the authors; it has been adopted because of the convenience of the system loss concept. Furthermore, one can effectively measure or interpret
data only for the scaled antenna situation. Even in communication practice
the concept of scaled (or constant gain) antennas is more convenient than that of constant-aperture antennas.

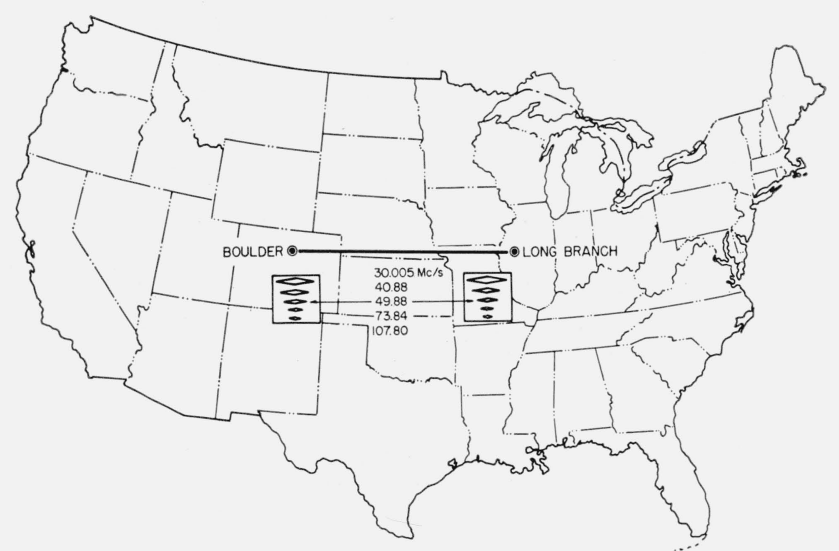

FIGURE 2. Experimental transmission path and frequencies. 
In the long-term recording program a detectorAGC circuit with an integration time constant of 12 sec was used; the recorder response was proportional to logarithm of the average signal voltage. For the short-term signal distributions encountered, comparison with fast-response recorder and totalizer measurements showed that median values given by the long-time constant record, scaled for periods of $5 \mathrm{~min}$ or so, corresponded with true median values of the distributions within a decibel. The use of integration considerably facilitated manual reduction of the records. Studies of short-term statistics were carried out separately.

The average signal voltage thus measured was equated to the rms value for computation of received power. Earlier tests [Boggs and Hekimian, 1953] showed that using the time average of the signal in this way may introduce up to $3 \mathrm{db}$ or more error in estimation of absolute power, but that little error is made in relative power ratios.

\section{Results}

\subsection{Short-Term Signal Statistics}

It was considered important to establish whether the statistical description of the short-term behaviors of the signals were sufficiently alike throughout the frequency band to warrant the assumption: (a) that the same propagation process was being observed at all frequencies and, to be assured (b) that the receiver was integrating signals alike at all frequencies. A number of fast-response recordings (receiver bandwidth $300 \mathrm{c} / \mathrm{s}$, recorder response $25 \mathrm{c} / \mathrm{s}$ ) were made of the fading signal received simultaneously at all frequencies.

Figure 3 shows cumulative distributions of signal envelopes at all frequencies, for typical daytime and nighttime conditions. The data shown were obtained from 10 -min sampling periods. It is seen from the figure that:

a. The distributions are similar at all frequencies.

b. The distributions approximate a Rayleigh distribution during the midday hours, and are somewhat peaked at the "high-signal" end during the late night and early morning hours.

Wheelon [1960] has recently discussed the amplitude distribution for a received signal containing effects of many meteor bursts, and obtains theoretical distributions similar to those shown for the night hours in figure 3.

Median crossings per second of positive slope, $N$, were obtained from these records. These fading rates were observed to vary over a wide range of values. For short periods (of the order of $30 \mathrm{sec}$ ) the value of $N$ may be from $0.2 \mathrm{c} / \mathrm{s}$ to $1.0 \mathrm{c} / \mathrm{s}$ at 30 $\mathrm{Mc} / \mathrm{s}$, with corresponding values of $1.0 \mathrm{c} / \mathrm{s}$ to $3 \mathrm{c} / \mathrm{s}$ at $108 \mathrm{Mc} / \mathrm{s}$. When signals are influenced by

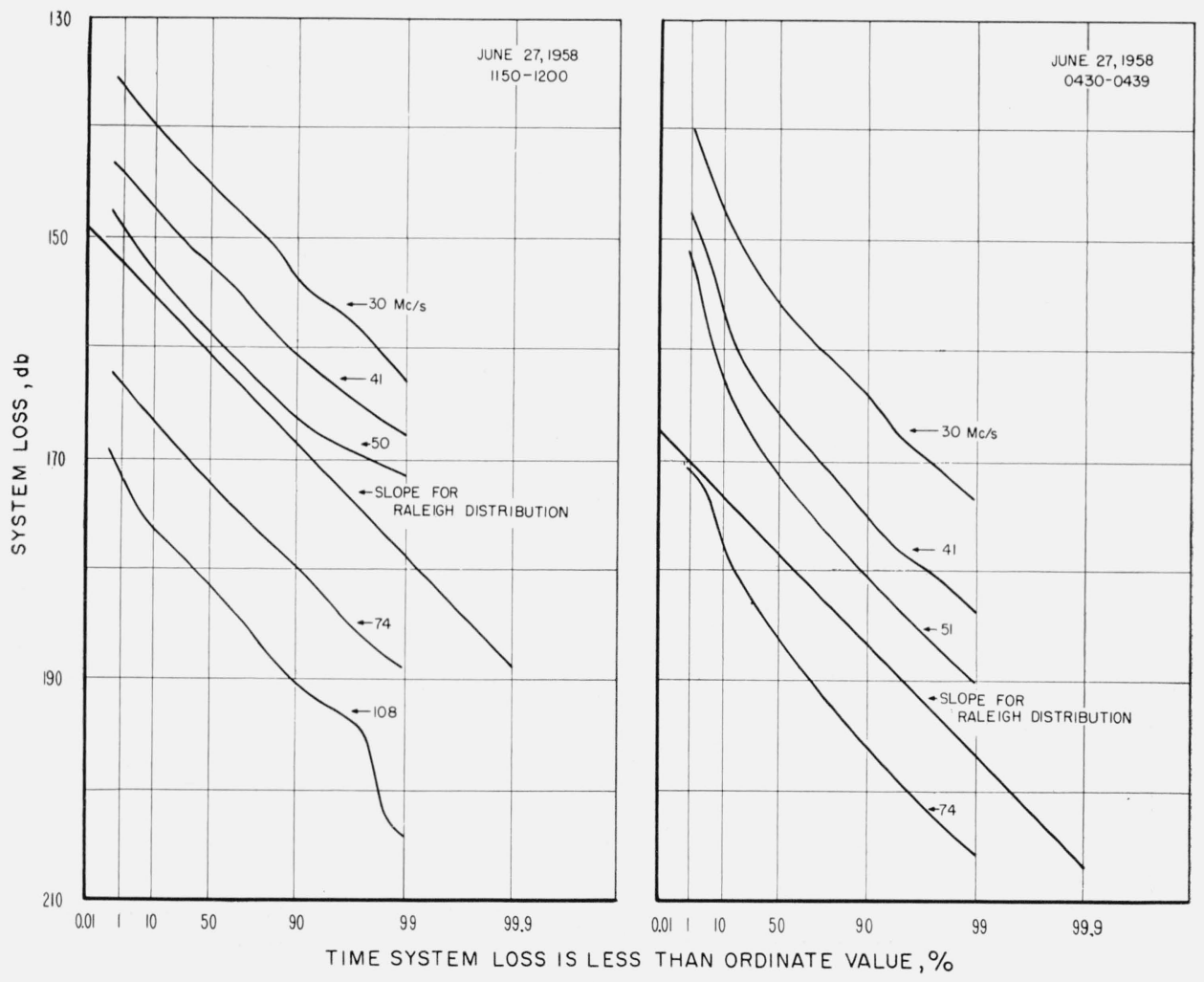

Figure 3. Cumulative distribution of fading envelope at five frequencies for ten minute periods; typical daytime and nighttime observations. 
sporadic- $E$ ionization, the fading rate may remain very low for several minutes. Figure 4 shows typical values of $N$ versus carrier frequency, averaged over 10 -min periods.

Most of the observations show $N$ proportional to carrier frequency to a power between $3 / 4$ and $5 / 4$. One would expect to observe linear dependence of fading rate on carrier frequency if the drift speed of the scattering region were high compared to the speed of change of structure of irregularities. Linear dependence of $N$ and good correlation of fading structure at all the frequencies would be expected if a single intense scatterer in motion, such as a meteor, were propagating the dominant signal, or if the signal were made up of an ensemble of such reflections. Departure from linear dependence of $N$ on frequency and poor frequency correlation almost certainly implies fading dominated by changing structure with in the scattering region.

\subsection{Hourly System Loss}

Continuous recording, using the receiver integration discussed in section 2 above, was carried out from October 1, 1958 through September 30, 1959. Hourly median values of the average signal trace were scaled for each hour of observation to obtain system loss values at the five frequencies.

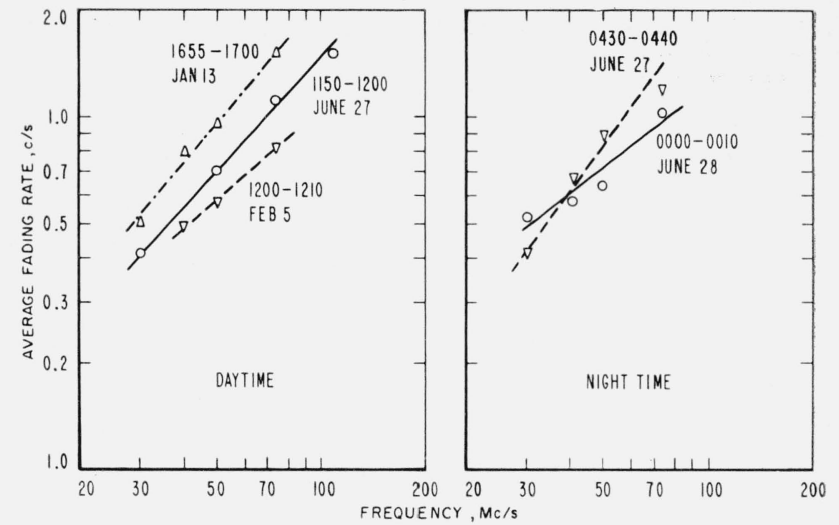

FiguRE 4. Observations of fading rate versus frequency; median crossings (one sign of slope) observed in ten minute period; typical daytime and nighttime observations.

Cumulative distributions of the hourly system loss values at all frequencies are plotted in figure 5 for 4 months of data representing conditions observed during summer, winter, and equinoxes. The solid curves represent the scatter signal data, with hours of identifiable sporadic- $E$ eliminated, and including some hourly values interpolated through short periods. One notes that the frequency dependence is a little lower at the weak-signal end of the distribution. This is discussed later.

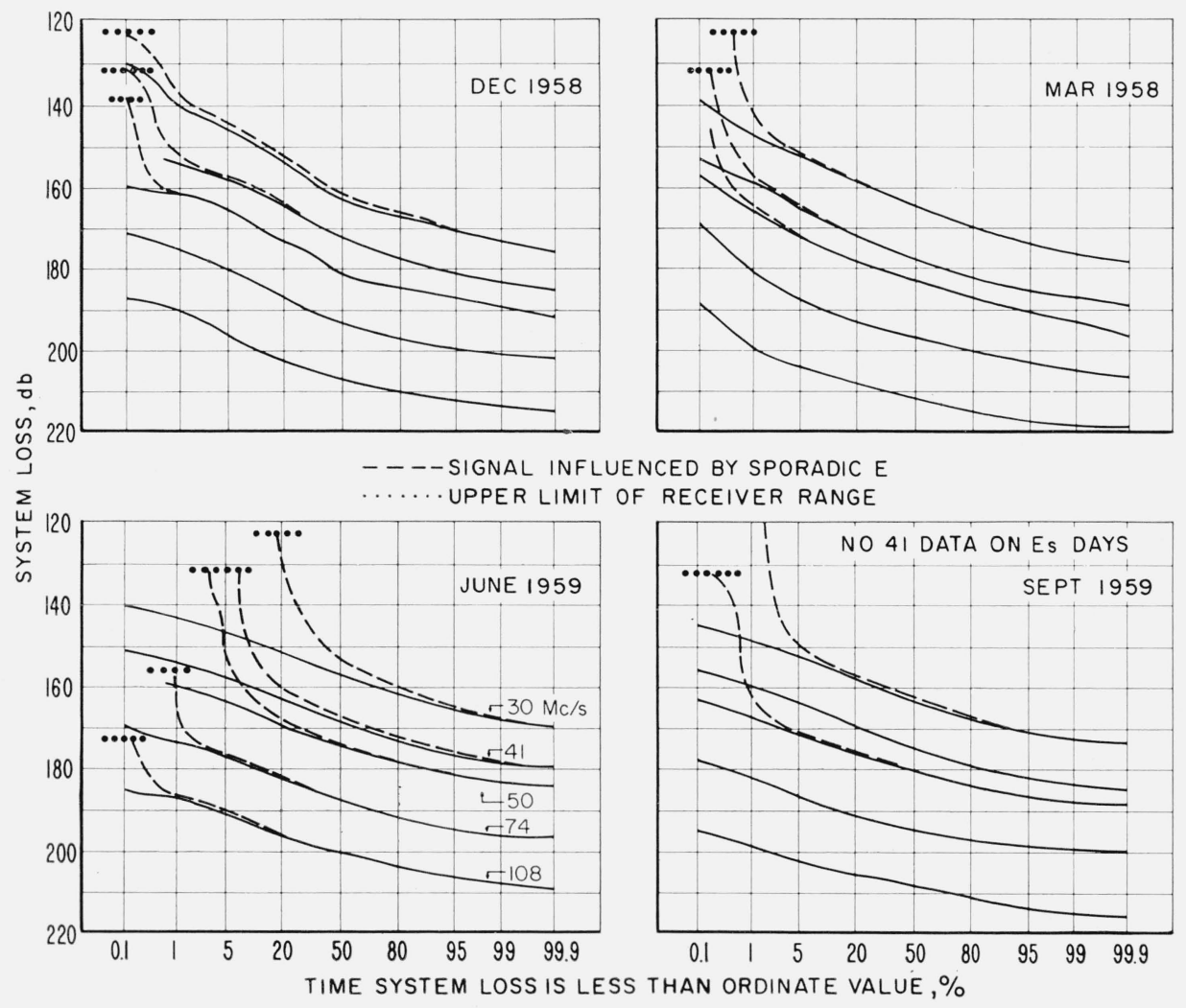

FIgURE 5. Monthly cumulative distributions of hourly values of system loss at five frequencies for months representative of winter, equinox and summer. 
Figure 6 shows the characteristic diurnal variation of the scatter signal at all frequencies for the same months as above. Monthly median values of the system loss for each hour of the day are plotted. One notes that the diurnal variation is consistently greater at lower frequencies than it is at higher frequencies. The effect, especially noticeable in the December and June curves, is progressive through the frequency range. This corresponds to diurnal variation of the frequency dependence which will be shown more explicitly later.

\subsection{Frequency Dependence of Hourly System Loss}

Using hourly median values of signal intensity observed at the five frequencies, linear least-squares regression lines of system loss in decibels versus log frequency were computed on an electronic computer for each hour's data for the 1-year observing period. The slope of this line is proportional to the hourly value of $n$. Standard error of $n$ was computed for each hour to check the goodness of fit of the straight line to the data. Median values of 0.30 for March 1959 and 0.34 for June 1959 are examples of the standard error.

Figure 7 is a representative plot of the observed hourly frequency dependence of system loss. Observed signal intensity at each frequency is plotted relative to that observed at the highest frequency, $108 \mathrm{Mc} / \mathrm{s}$. The lines are indicative of individual hourly observations but the plotted points in the figure are actually median values of all hourly observations in July 1959, for hours near midday and

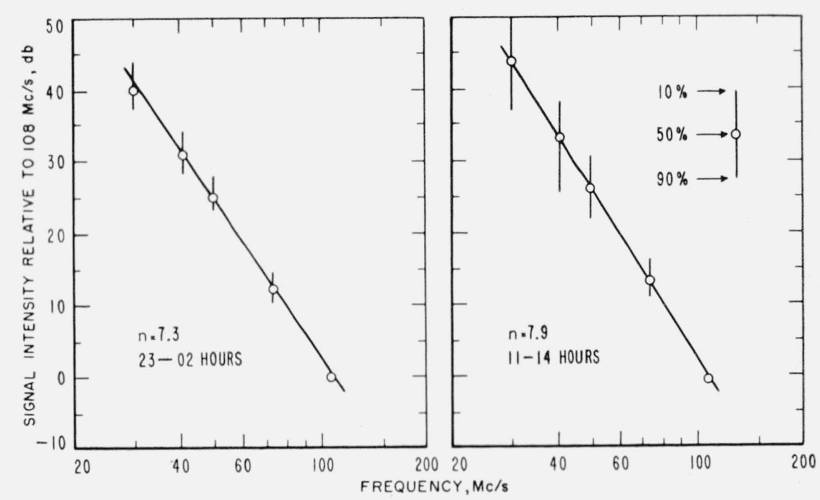

Figure 7. Frequency dependence of system loss; observed signal intensity at each frequency plotted relative to that observed at $108 \mathrm{Mc} / \mathrm{s}$; values shown in plot represent all hourly values near midday and midnight during July 1959.

near midnight. The spread of hourly observations is indicated by the vertical lines through the points, the extremities of which represent the values observed 10 and 90 percent of the time. Because of the goodness of the fit of the points to straight lines at all hours, the spread shown is also nearly representative of the computed regression lines.

The actual correspondence of results with this "straight line" law is the most striking departure of the present results from earlier work. Though $n$ varies from about 6 to 11 , with characteristic diurnal and seasonal variation, received power is always closely represented by the strict power law, $p_{t} / p_{a} \propto f^{n}$,

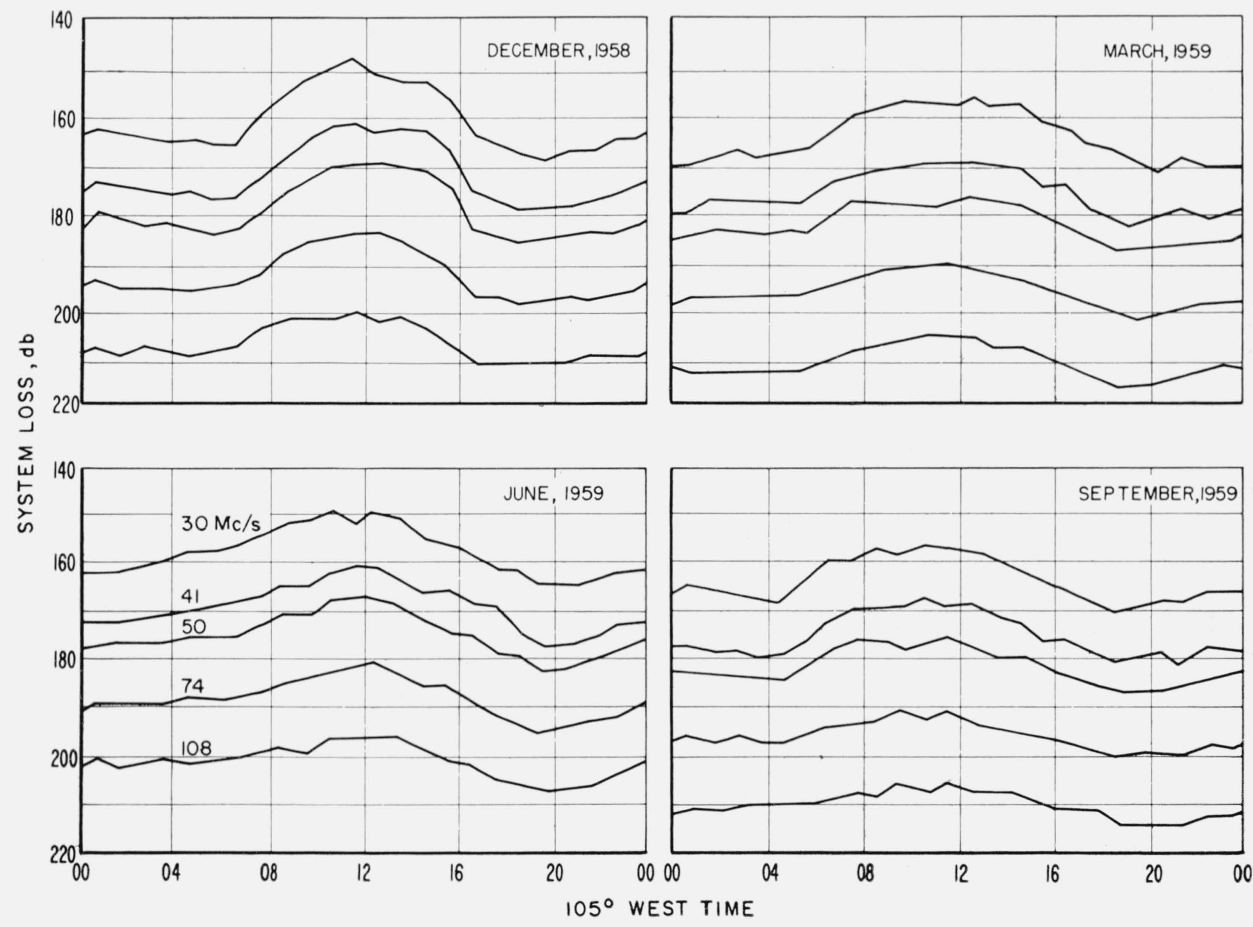

FIgURE 6. Monthly median plots of hourly values of system loss, showing diurnal variation at five frequencies in winter, equinox and summer. 
over the range of frequencies observed. The earlier NBS results [Bailey, Bateman and Kirby, 1955] had been interpreted as giving a lower value of $n$ for the lower part of the frequency range (28 to $50 \mathrm{Mc} / \mathrm{s}$ ) than was obtained for the higher part (50 to $108 \mathrm{Mc} / \mathrm{s})$. The two pairs of frequencies had not been observed simultaneously, and later studies of the siting and antenna characteristics also raised some questions about the idealness of the radiation patterns. It is felt that the careful siting and calibration of antennas and power in the present experiment, together with the feature of simultaneous comparison at frequencies throughout the whole range, fully justifies revision of the earlier conclusion and adoption of the idea of a strict power law. It thus appears valid to express the turbulent spectrum function $S(k)$ as a power relationship $k^{-(n-2)}$.

The straight-line law also has implications as to absorption effects. During the course of the VHF measurements, attenuation data over the same path were obtained from a continuous $5 \mathrm{Mc} / \mathrm{s}$ transmission. Sufficient sensitivity was achieved to permit continuous day and night observation at the single frequency, so that information was obtained on diurnal and seasonal variation of nondeviative $D$-region attenuation. The observed values of attenuation at $5 \mathrm{Mc} / \mathrm{s}$ were extrapolated to $\mathrm{VHF}$ by the relation

$$
A d b \propto \frac{1}{\left(f_{H}+f\right)^{2}} .
$$

This indicated that if the scatter signal suffered full $D$-region attenuation, the noon values at $30 \mathrm{Mc} / \mathrm{s}$ would be reduced by 3 to $4 \mathrm{db}$ and noticeable curvature would be introduced into the scaling law. Alter- natively, if the observed straight line law included the effect of daytime absorption, then the absence of absorption at night would introduce curvature. But the experimental results gave a greater value of $n$ by day than at night and a straight line at all times except during abnormal absorption events such as are associated with solar flares. It appears that under normal conditions the VHF scatter signal does not encounter measurable attenuation and therefore must not traverse a significant part of the absorbing region.

\subsection{Diurnal and Seasonal Variation of Frequency Dependence}

Figure 8 shows that the value of $n$, varying over a range from about 6 to 11 , is characteristically maximum during the daytime. This figure shows values of $n$ exceeded 90 percent, 50 percent, and 10 percent of the days for each of four months representing all seasons. Diurnally $n$ usually varies \pm 1 , though July shows less.

Figure 9 shows systematic seasonal variation of $n$. The median of all daytime values (hours 0900 to 1400) and nighttime values (hours 2100 to 0200 ) is plotted for each month; despite the spread of values in each month, indicated by the vertical lines, the seasonal variation seems clear. A summer minimum is distinct and there is a suggestion in the daytime values of a secondary winter minimum.

It is of interest to inquire whether the summer minimum represents absorption effects; i.e., whether greater summer $D$-region absorption tends to reduce the net frequency exponent of the scatter signals.
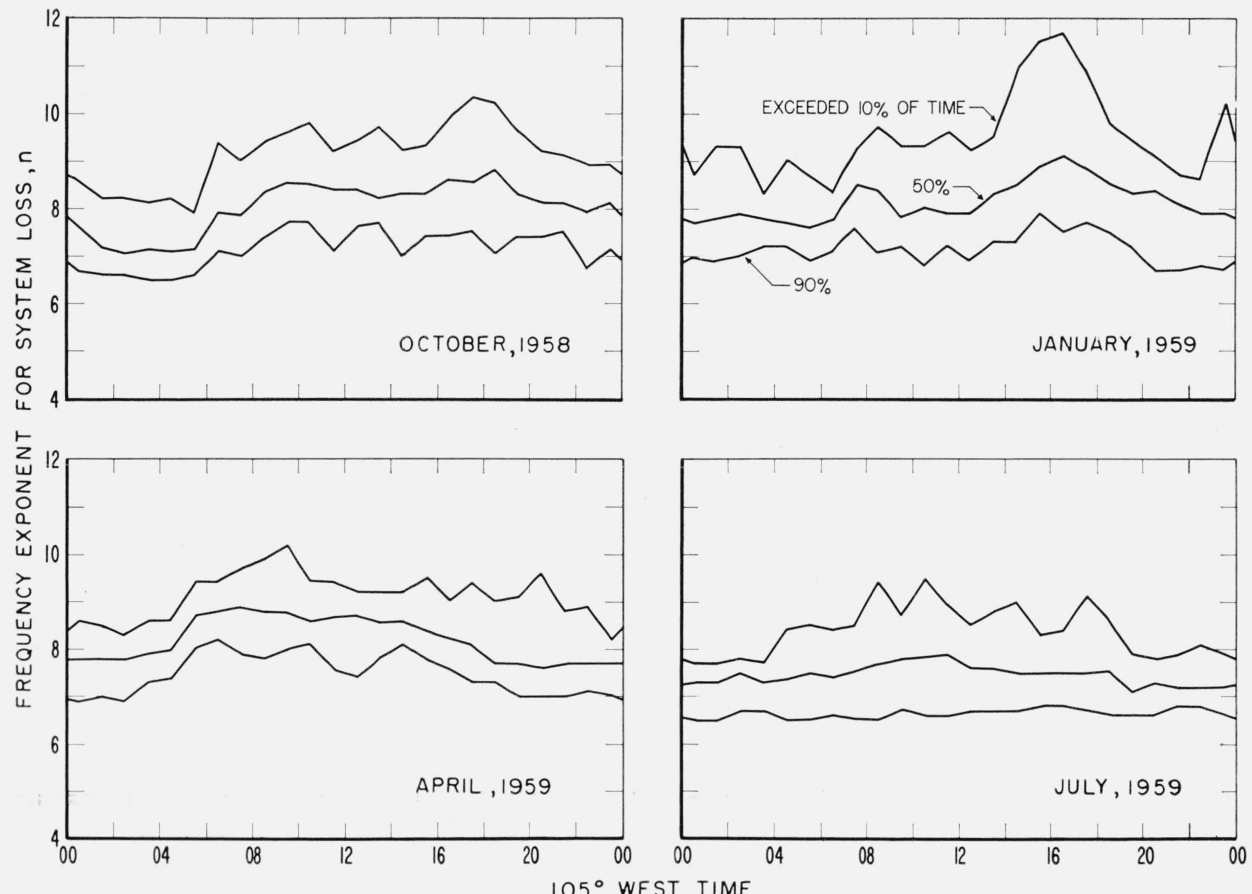

Figure 8. Diurnal variation of $n$ during four seasons. 


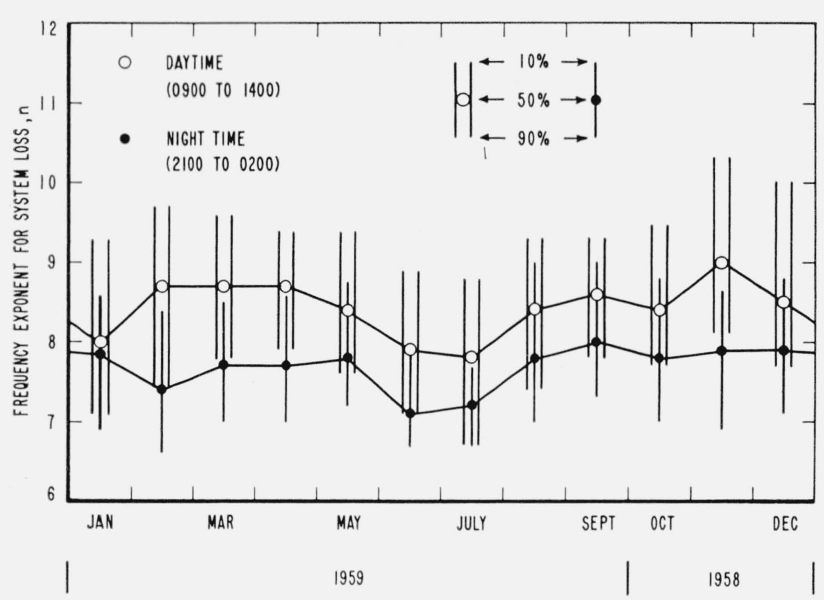

Figure 9. Seasonal variation of $n$ for daytime and nighttime observations.

However, the seasonal depression of values of $n$ is about as great at night as during the day, and $n$ is greater during the day. These observations again tend to rule out absorption as a factor. It seems more likely that the diurnal and seasonal variation of $n$ represents changing relative roles of meteoric reflections and turbulence.

A frequency exponent of 7 has been estimated for scatter signals that are composed entirely of meteoric echoes [Villard, Eshleman, Manning, and Peterson, 1955]. Thus, the value of 7 could be expected to prevail at times when the signal is mostly dominated by meteoric reflections. Higher values are provided
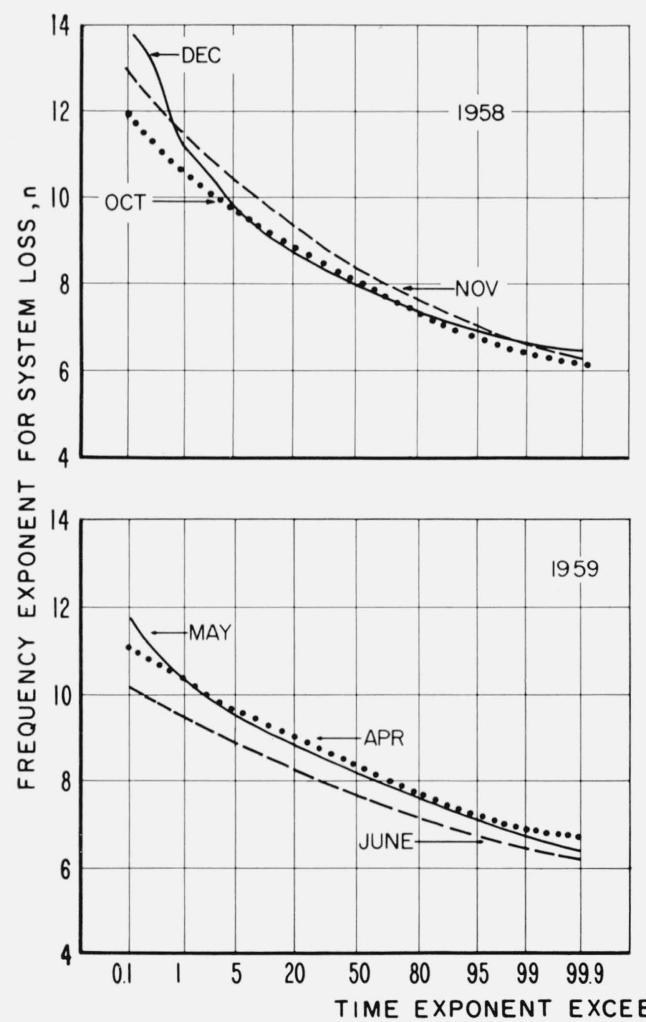

FIgURE 10. Cumulative distribution of values of $n$ observed during each month of the year. not suggested this. 9.7 .

\subsection{Weak-Signal Conditions} values are obtained:
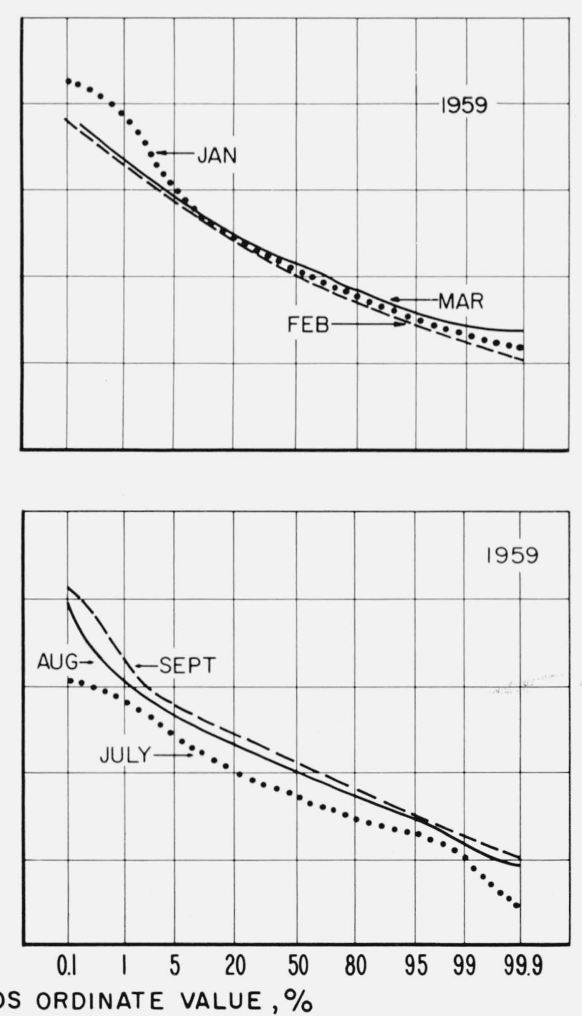

by turbulence theory. Comparison of $n$ for broadbeam and narrow-beam systems discussed in 3.6 also strengthens the idea that variation of the relative role of meteor reflections may account for part of the diurnal and seasonal variation of $n$. It is also possible that the $S(k)$ for turbulence varies with time of day and season, though the theories have

Figure 10 shows cumulative distributions of hourly values of $n$ observed during each month of the program. For the year, the median value of all observations of $n$ was 8 . Ninety-five percent of the observations exceeded 6.9 and five percent exceeded

It is of interest to the design engineer to know the frequency dependence of system loss during conditions of weakest signals, which generally govern the limitations of the system capacity. Values of $n$ associated with signal levels exceeded large percentages of the time can be obtained by comparing the cumulative distributions of system loss for a year's observations at each frequency. The following

\begin{tabular}{r|c}
\hline \hline Value of $n$ & $\begin{array}{c}\text { Obtained for system loss cor- } \\
\text { responding to signal level } \\
\text { exceeded: }\end{array}$ \\
\cline { 2 - 2 } 8.1 & $50 \%$ of time \\
7.6 & $90 \%$ \\
7.4 & $99 \%$ \\
\hline
\end{tabular}


A value $n=7.4$ may thus be taken for engineering computations concerned with weak signal conditions. If galactic noise is limiting at the receiver, the net signal-to-noise ratio may be taken to vary as $\sim f^{-5}$, allowing for the frequency dependence of the noise.

\subsection{Effect of Beam Width}

A parallel experimental system, using horizontal Yagi transmitting and receiving antennas with azimuthal beam widths of $56^{\circ}$, was available over the same path as the narrow-beam systems. This broad-beam system provided the opportunity for simultaneous frequency-dependence observations at two frequencies, near 30 and $50 \mathrm{Mc} / \mathrm{s}$.

Figure 11 compares monthly median values of $n$ at all hours of the day for the two systems for September 1959. The broad-beam system values of $n$ range from $\frac{1}{2}$ to 1 lower than the narrow-beam values. The diurnal variation of $n$ is more pronounced for the broad-beam system, and distinct minimums are observed during early evening and early morning hours.

These results are interpreted as additional evidence that a lower value of $n$ is associated with the meteoric contribution more effectively than with the narrowbeam system directed along the Great Circle, especially during early morning and evening hours, for geometrical reasons discussed in the literature. More noticeable absorption effects in the broad-beam system might also tend to reduce the effective exponent, as transmission off the Great Circle must traverse a longer path in the $D$-region. These absorption effects have manifest themselves during at least one intense absorption event associated with a solar flare, when signals in the broad-beam system were attenuated much more severely than the narrow-beam system signals.

\subsection{Abnormal $D$ Ionization}

A number of observations were made during abnormal events such as daylight occurrences of sudden ionospheric disturbances associated with solar flares, and magnetic disturbances.

During SID's attenuation was characteristically observed at the lowest frequencies, and an increase

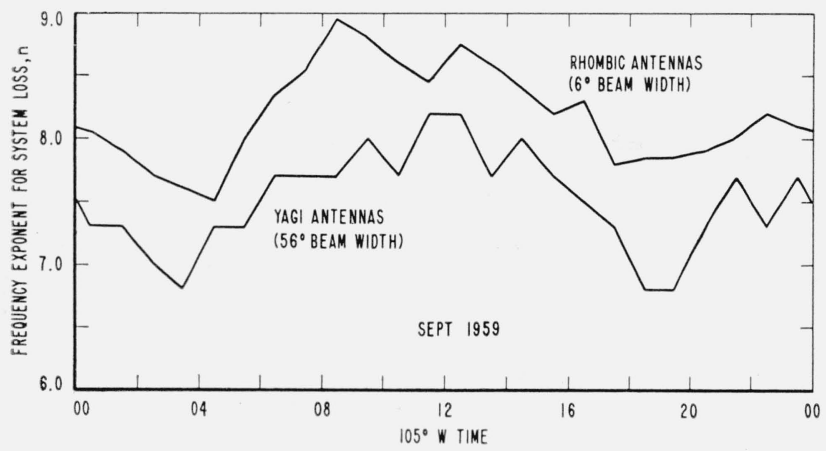

Figure 11. Comparison of values and diurnal variation of $n$ for narrow and broad beam systems (measurements made simultaneously). of scattered signal intensity was observed at the highest frequencies. Attenuation of as much as $30 \mathrm{db}$ has been observed at $30 \mathrm{Mc} / \mathrm{s}$, but the signal never dropped below the threshold of observability. Usually, the attenuation was overtaken by increased scattering to produce a net enhancement within 10 to $30 \mathrm{~min}$. Attenuation is rarely observed at 50 $\mathrm{Mc} / \mathrm{s}$; usually an enhancement is noticeable.

Figure 12 shows signal behavior at five frequencies during an SID of March 29, 1958. Absorption is severe at $30 \mathrm{Mc} / \mathrm{s}$, and is noticeable at $41 \mathrm{Mc} / \mathrm{s}$. Note that a signal enhancement is observed at all frequencies, somewhat greater and later at the lower frequencies. The initial rise in signal intensity at $108 \mathrm{Mc} / \mathrm{s}$ is essentially simultaneous with the onset of the absorption at $30 \mathrm{Mc} / \mathrm{s}$. As time progresses increased scattering develops at the lower frequencies, eventually overtaking the attenuation effects at $30 \mathrm{Mc} / \mathrm{s}$.

It is of interest that during the same event for which the narrow-beam system data is given in figure 12 , approximately twice as much attenuation (in decibels) was observed on the broad-beam Yagi system at $30 \mathrm{Mc} / \mathrm{s}$, and transmission at frequencies as high as $74 \mathrm{Mc} / \mathrm{s}$ suffered noticeable attenuation. This is attributed to the greater path length through the absorbing $D$-region for the energy propagated off the Great Circle; it is also likely that dependence of the broad beam propagation on meteoric echoes at higher ionospheric heights than the absorbing $D$ region tends to increase the effective attenuation.

\section{Summary}

The frequency dependence of system loss has been found to follow a strict power law $p_{t} / p_{a} \propto f^{n}$ over the frequency range of the experiment, 30 to 108 $\mathrm{Mc} / \mathrm{s}$, at all times except during abnormal ionospheric absorption. Thus, it seems the spectrum of turbulence $S(k)$ may be characterized by an $\lambda^{n-2}$ dependence. Figure 13 compares the distribution of $n$ with theoretical values from Wheelon's [1960a] summary of spectral models.

The value of $n$ varies diurnally and seasonally; 90 percent of all observations lie in the range 7 to

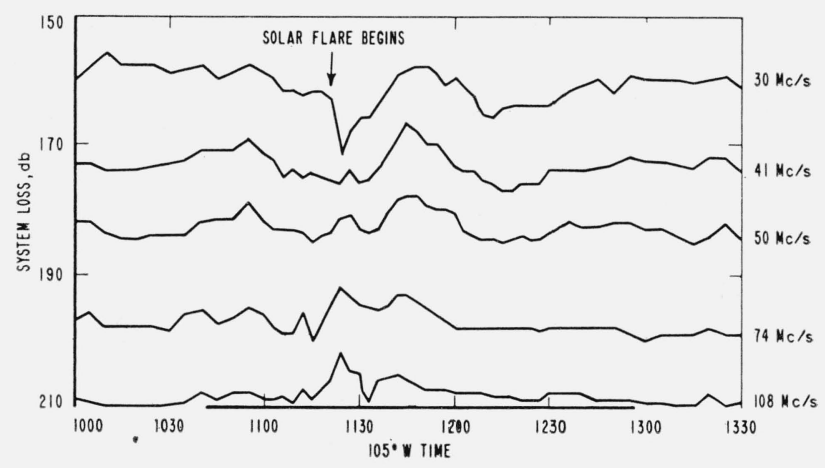

Figure 12. Signal behavior at five frequencies during an intense ionospheric absorption event associated with a solar flare, March 29, 1958. 
